TRANSACTIONS OF THE

AMERICAN MATHEMATICAL SOCIETY

Volume 353, Number 9 , Pages 3760-3787

$\mathrm{S} 0002-9947(01) 02832-\mathrm{X}$

Article electronically published on May 4, 2001

\title{
BEREZIN TRANSFORM ON REAL BOUNDED SYMMETRIC DOMAINS
}

\author{
GENKAI ZHANG
}

\begin{abstract}
Let $\mathbb{D}$ be a bounded symmetric domain in a complex vector space $V_{\mathbb{C}}$ with a real form $V$ and $D=\mathbb{D} \cap V=G / K$ be the real bounded symmetric domain in the real vector space $V$. We construct the Berezin kernel and consider the Berezin transform on the $L^{2}$-space on $D$. The corresponding representation of $G$ is then unitarily equivalent to the restriction to $G$ of a scalar holomorphic discrete series of holomorphic functions on $\mathbb{D}$ and is also called the canonical representation. We find the spectral symbol of the Berezin transform under the irreducible decomposition of the $L^{2}$-space.
\end{abstract}

\section{INTRODUCTION}

The main purpose of the present paper is to calculate the spectral symbol of the Berezin transform on real bounded symmetric domains. To explain our results and motivations we let $\mathbb{D}$ be the unit disk in the complex plane with the Lebesgue measure $d m(z)$. We consider the weighted Bergman space $H^{\nu}(\nu>1)$ of holomorphic functions on $\mathbb{D}$ square integrable with respect to the measure $\left(1-|z|^{2}\right)^{\nu-2} d m(z)$. It has up to some positive constant the reproducing kernel $K_{w}(z)=K(z, w)=$ $(1-z \bar{w})^{-\nu}$. Moreover the group $G_{c}=S U(1,1)$ of fractional transformations of $\mathbb{D}$ acts on the space $H^{\nu}$ via $f(z) \mapsto f(g z) g^{\prime}(z)^{\frac{\nu}{2}}$ and it forms an irreducible unitary (projective) representation. Consider the subgroup $S O(1,1)$ consisting of transformations of the form $z \mapsto \frac{a z+b}{b z+a}$ with $a, b \in \mathbb{R}$ and $a^{2}-b^{2}=1$. Thus it is of interest to study the irreducible decomposition of the weighted Bergman space under the subgroup $G=S O(1,1)$. For that purpose we consider the unit interval $D=\mathbb{D} \cap \mathbb{R}=(-1,1)$ as a trivial symmetric space $G / K=S O(1,1) /\{ \pm 1\}$ and the restriction of holomorphic functions in $H^{\nu}$ to the interval $D$. More precisely, consider $R: H^{\nu} \rightarrow C^{\infty}(D)$,

$$
R f(x)=f(x)\left(1-x^{2}\right)^{\frac{\nu}{2}}, \quad x \in D .
$$

Let $L^{2}\left(D, d \mu_{0}\right)$ be the $L^{2}$ space on $D$ with the $S O(1,1)$-invariant measure $d \mu_{0}(x)=$ $\frac{d x}{\left(1-x^{2}\right)}$, whose decomposition under $S O(1,1)$ can be done via the Mellin transform (see below). The restriction $R$ is a bounded operator from $H^{\nu}$ into the space $L^{2}\left(D, d \mu_{0}\right)$ with dense image, and intertwines the respective actions of $S O(1,1)$,

Received by the editors January 16, 2000 and, in revised form, October 10, 2000.

2000 Mathematics Subject Classification. Primary 22E46, 43A85, 32M15, 53C35.

Key words and phrases. Real bounded symmetric domains, Jordan triples, Siegel domains, Berezin transform, invariant differential operators, unitary representations of Lie groups, irreducible decomposition.

Research supported by the Swedish Natural Sciences Research Council (NFR). 
see Section 1. To get a unitary intertwining operator we consider the polar decomposition of $R, R=|R| U$. Thus $U=|R|^{-1} R$ is a unitary intertwining operator from $H^{\nu}$ onto $L^{2}\left(D, d \mu_{0}\right)$, with $|R|^{2}=R R^{*}$ given by

$$
B_{\nu} f(x)=\frac{\Gamma(\nu)}{2^{\nu-1} \Gamma\left(\frac{\nu}{2}\right)^{2}} \int_{D} f(y) \frac{\left(1-x^{2}\right)^{\frac{\nu}{2}}\left(1-y^{2}\right)^{\frac{\nu}{2}}}{(1-x y)^{\nu}} d \mu_{0}(y)
$$

which we call the Berezin transform on $D$.

Now the $L^{2}\left(D, d \mu_{0}\right)$, after performing the Cayley transform $x \mapsto \frac{1-x}{1+x}$ mapping $D$ to the half line $\mathbb{R}^{+}=(0, \infty)$, can be decomposed under $S O(1,1)$ via the Mellin transform,

$$
f(x) \mapsto \hat{f}(\lambda)=\int_{0}^{\infty} f(x) e_{\lambda}(x) \frac{d x}{x},
$$

with $e_{\lambda}(x)=x^{\lambda}$ and $\lambda \in i \mathbb{R}$. On the half real line it becomes

$$
B_{\nu} f(x)=\frac{\Gamma(\nu)}{\Gamma\left(\frac{\nu}{2}\right)^{2}} \int_{0}^{\infty} f(y) \frac{x^{\frac{\nu}{2}} y^{\frac{\nu}{2}}}{(x+y)^{\nu}} \frac{d y}{y}
$$

on the space $L^{2}\left(\mathbb{R}^{+}, \frac{d x}{x}\right)$. $B_{\nu}$ is then a function of the self-adjoint operator $L=i x \frac{d}{d x}$. In terms of the eigenfunction $e_{\lambda}(x), B_{\nu} e_{\lambda}=b_{\nu}(\lambda) e_{\lambda}$,

$$
b_{\nu}(\lambda)=\frac{\Gamma\left(\frac{\nu}{2}+\lambda\right) \Gamma\left(\frac{\nu}{2}-\lambda\right)}{\Gamma\left(\frac{\nu}{2}\right)^{2}}
$$

$b_{\nu}(\lambda)$ is obtained by calculating the integral $B_{\nu} e_{\lambda}(x)$ at $x=1$. Thus $B_{\nu}=b_{\nu}(i L)$.

One can formulate the above problem for any real bounded symmetric domain, where the operator $L$ is replaced by a system of generators. The intertwining operator $R$ for a general bounded symmetric domain was introduced earlier in [12]. The main purpose of the present paper is to find the symbol $b_{\nu}$. Moreover, we prove that the Berezin transform defines a bounded operator on the $L^{p}$-space $(1 \leq$ $p \leq \infty)$ on $D$ with the invariant measure when $\nu$ is in a certain interval, and thus justifies the operator theoretic meaning of the symbol. In particular, this proves the boundedness of the intertwining operator $R$ from the analytic continuation of the holomorphic discrete series to the $L^{2}$-space on $D$. The exact interval of $\nu$ depends on different root systems. For type $B C$ and $C$ the interval of $\nu$ is such that $H^{\nu}$ is a discrete series of $\mathbb{D}$; however, for other types of domains the range of $\nu$ is larger than the holomorphic discrete series, but still above the reducible points.

Before explaining our methods of calculation we make some general remarks and put our results into perspective. The problem of finding the irreducible decomposition of a unitary representation $(\pi, H)$ of a Lie group $H$ under a subgroup $G \subset H$ has been studied for a long time, both in mathematical physics and in representation theory, and is called the "Branching rule". We can also put the Berezin transform on the unit disk $\mathbb{D}$, originally introduced by Berezin [2] for any complex Kähler manifold, in this context. Consider the tensor product $H^{\nu} \otimes \overline{H^{\nu}}$ of the weighted Bergman space on $\mathbb{D}$ with its conjugate realized as space of functions $f(z, w)$ holomorphic in $z$ and anti-holomorphic in $w$. It forms a unitary representation of the group $G_{c} \times G_{c}$. Then $G_{c}$ can be considered as the subgroup $\left\{(g, g) ; g \in G_{c}\right\}$ of $G_{c} \times G_{c}$. To find the irreducible decomposition of the tensor product under $G_{c}$ we consider also the restriction map of the function $f(z, w)$ to its diagonal $f(z, z)$; 
more precisely, $R f(z)=f(z, z)\left(1-|z|^{2}\right)^{\nu}$. In this way we also obtain the Berezin transform on $\mathbb{D}, B_{\nu}=R R^{*}$,

$$
B_{\nu} f(z)=\int_{\mathbb{D}} f(w) \frac{\left(1-|z|^{2}\right)^{\nu}\left(1-|w|^{2}\right)^{\nu}}{\left|(1-z \bar{w})^{\nu}\right|^{2}} \frac{d m(w)}{\left(1-|w|^{2}\right)^{2}} .
$$

See [13]. The symbol of the Berezin transform for a general bounded symmetric domain was found by Unterberger and Upmeier [17]. The spectral symbol of the Berezin transform then gives the spectral decomposition of the tensor product $H^{\nu} \otimes$ $\overline{H^{\nu}}$ of $G_{c}$ for large $\nu$. (See also [21] where the tensor product $H^{\nu} \otimes \overline{H^{\kappa}}$ for different $\nu$ and $\kappa$ is considered and the corresponding Berezin symbol is calculated.) The exact formula plays a decisive role in decomposing the tensor product for small $\nu$. Interestingly there appears discrete parts in the decomposition; cf 14 for the case of the unit disk $\mathbb{D}=S U(1,1) / U(1)$ and [6] for the matrix ball $S U(p, q) / S(U(p) \times$ $U(q))$.

We proceed to explain our methods of calculation. We will use the idea of Unterberger and Upmeier [17, applying the theory of Jordan triples and Gindikin Gamma function. Let $V=V_{2} \oplus V_{1}$ be the Peirce decomposition of $V$ with respect to a maximal tripotent $e$. The Jordan algebra $V_{2}$ has further a Cartan decomposition $V_{2}=A \oplus B$. The bounded domain $D=G / K$ can be realized as a real Siegel domain in $V$. The Harish-Chandra $e_{\underline{\lambda}}$-function has a rather explicit form, in terms of the determinant function on $V_{2}$; see (2.6). The symbol $b_{\nu}(\underline{\lambda})$ is then an integral on the Siegel domain. The integral reduces further to one integration on $V_{1}$, one on $B$ and one on the symmetric cone $\Omega$ in $A$. In the case of the complex Siegel domains, $B=i A$ and the integration on $B$ can be obtained directly by the Laplace transform on $\Omega$. However, for real domains $B$ is not very much related to $A$, and we calculate the integral by using a method of Shimura [16], writing the integral as one on the cone $\Omega$ in $A$; see Proposition 3.1.

The symbols of the Berezin transform have also been calculated previously by Neretin 10 for classical domains, and by van Dijk and Hille 18 for classical rank one domains. As we will see in this paper, the integrals involved are generalization of the classical Beta-integral. For certain special cases they were also studied earlier by Hua [7].

The Berezin transform formally defines a positive operator, namely $\left(B_{\nu} f, f\right) \geq 0$ for all $f \in C_{0}^{\infty}(D)$. Thus the problem of finding the irreducible decomposition of $H^{\nu}$ under $G$ can also be formulated as finding the decomposition defined by the positive definite kernel $\frac{\left(1-|z|^{2}\right)^{\nu}\left(1-|w|^{2}\right)^{\nu}}{\left|(1-z \bar{w})^{\nu}\right|^{2}}$. In this context it is also called canonical representation, introduced and named by Vershik, Gel'fand and Graev 20].

Finally, we remark that even though our main goal is the symbol of the Berezin transform, some results in this paper are of independent interests. In particular, our Propositions 3.1 and 4.1 generalize the formula (2.13) in [16. The exact value of those integrals is critical in studying the analytic continuation of Bessel functions and Eisenstein series; see loc. cit. We hope to study applications of our results to Bessel functions in future publications.

The paper is organized as follows. In Section 1 we introduce real bounded symmetric domains and the Berezin transform. In Section 2 we consider the HarishChandra $e$-function defined abstractly in terms of the Iwasawa decomposition $G=$ $N A K$. We give explicit formulas for positive root vectors, and thus give a formula of the $e$-function in terms of the conical functions. The symbol of the Berezin transform is calculated in Sections 3, 4 and 5 for the different root systems. In 
Section 6 we prove the $L^{p}$-bounded properties of the Berezin transform, therefore justifying the operator theoretic meaning of the symbol $b_{\nu}$ of the Berezin transform.

After this paper was finished the author received a preprint of [19 by van Dijk and M. Pevzner, where they also found the symbol for real tube domains using slightly different methods.

Acknowledgement. I would like to thank Professor Goro Shimura for drawing his paper [16 to my attention and for his encouragement. I am grateful to Professors Yurii Neretin and Bent Ørsted for some helpful discussions.

For the reader's convenience we list the main symbols used in the paper:

1. $\mathbb{D}=G_{c} / K_{c}$, an irreducible bounded symmetric domain in a complex vector space $V_{\mathbb{C}}=\mathbb{C}^{n}, V$ a real form of $V_{\mathbb{C}}$ and $D=\mathbb{D} \cap V$ a real bounded symmetric domain in $V$;

2. $\mathfrak{g}=\mathfrak{k}+\mathfrak{p}$, the Cartan decomposition of $\mathfrak{g}, \mathfrak{a} \subset \mathfrak{p}$ a maximal abelian subspace of $\mathfrak{p}$;

3. $D(z, \bar{w}) v=\{z \bar{w} v\}$, the Jordan triple product;

4. $B(z, w)=I-D(z, \bar{w})+Q(z) Q(\bar{w})$, the Bergman operator;

5. $(z, w)$, normalized scalar product on $V$ so that a minimal tripotent has norm 1 ;

6. $p$ the genus of domain $\mathbb{D}$ and $p_{0}$ the genus of $D$ (see Definition 2.2);

7. $h(z, w)$ an irreducible polynomial on $V_{\mathbb{C}} \times \overline{V_{\mathbb{C}}}$, and $\operatorname{det} B(z, w)=h(z, w)^{p}$;

8. $V=V_{2} \oplus V_{1}$ Peirce decomposition with respect to a maximal tripotent $e$, $V_{2}=A \oplus B$ the Cartan decomposition of $V_{2}$ with respect to the involution $z \mapsto Q(e) \bar{z} ;$

9. $\Delta$ the determinant function of $V_{2}$ and $\delta$ the determinant function of $A$;

10. $\mathcal{S}$ the Siegel domain realization of $D$.

1. REAL BOUNDED SYMMETRIC DOMAINS AND RESTRICTION OF HOLOMORPHIC FUNCTIONS

We recall first some preliminary results on real bounded symmetric domains and fix notation. Our presentation is mainly based on Loos 9 .

Let $\mathbb{D}=G_{c} / K_{c}$ be an irreducible bounded symmetric domain in a complex vector space $V_{\mathbb{C}}=\mathbb{C}^{n}$. The space $V$ has then a Jordan triple structure. We denote $\{x \bar{y} z\}$ the Jordan triple product. Let $V$ be a real form of $V_{\mathbb{C}}$ and $\tau$ be the conjugation with respect to $V$. Suppose $\tau(\mathbb{D})=\mathbb{D}$, namely, $\tau$ fixes the bounded symmetric domain. Then the real form $D=\mathbb{D} \cap V$ will be called a real bounded symmetric domain. In this case the triple product $D(x, \bar{y}) z=\{x \bar{y} z\}$ restricted on $V$ defines also a triple product on $V$. A complete list of real bounded symmetric domains $D$ is given in 9]. As a Riemannian symmetric space, $D=G / K$, where $G$ is the subgroup of $G_{c}$ consisting biholomorphic transformations of $\mathbb{D}$ which keep $D$ invariant. The coset space $G_{c} / G$ is called a causal symmetric space, a complete list of the pairs $\left(G_{c}, G\right)$ can be found in e.g. [11, [5] and [6].

We describe briefly some algebraic and geometric structures of the domain $D$.

Let $\mathfrak{g}=\mathfrak{p} \oplus \mathfrak{k}$ be the Cartan decomposition of the Lie algebra $\mathfrak{g}$ of $G$. The Lie algebra of $G_{c}$ will be realized as completely integrable holomorphic vector fields on $\mathbb{D}$. The elements in $\mathfrak{g}$ can then be written as $\mathfrak{g}=\left\{\xi_{v}(z)=v-Q(z) \bar{v} ; v \in V\right\}$ where $Q(z) \bar{v}=\frac{1}{2}\{z \bar{v} z\}$ is the quadratic operator.

We fix a $K$-invariant inner product $(\cdot, \cdot)$ on $V$ so that a minimal tripotent has norm 1 , and let $\langle\cdot, \cdot\rangle$ be the corresponding Hermitian inner product on $V_{\mathbb{C}}$. (A 
minimal tripotent of $V_{\mathbb{C}}$ has norm $\frac{\sqrt{2}}{2}$ if the root system is of Type BC or C, otherwise it is of norm 1; see below.) We let $d z$ be the corresponding Lebesgue measure.

Denote further $B(x, \bar{y})$ the Bergman operator

$$
B(x, y)=1-D(x, \bar{y})+Q(x) Q(\bar{y}) .
$$

The Bergman kernel of $\mathbb{D}$ is up to a constant $h(z, w)^{-p}$ where $h(z, w)$ is an irreducible polynomial and $p$ is the genus of $\mathbb{D}$. For simplicity we write $h(z)=h(z, z)$. The Bergman metric of $\mathbb{D}$ is

$$
\left\langle B(z, z)^{-1} u, v\right\rangle .
$$

The domain $D$ is a totally real and totally geodesic submanifold of $\mathbb{D}$ and its Riemannian metric is the above Bergman metric; with this metric it is also a Riemannian symmetric space, $D=G / K$. The $G$-invariant measure on $D$ is

$$
h(z)^{-\frac{p}{2}} d m(z),
$$

and $G$ acts unitarily on $L^{2}\left(D, h^{-\frac{p}{2}} d m\right)$ via change of variables.

Consider the weighted Bergman space $H^{\nu}(\mathbb{D})$ of holomorphic functions on $\mathbb{D}$ so that

$$
\int_{\mathbb{D}}|f(z)|^{2} h(z)^{\nu} \frac{d m(z)}{h(z)^{p}}<\infty
$$

The group $G_{c}$ acts unitarily on $H^{\nu}(\mathbb{D})$ via

$$
\pi_{\nu} f(z)=J_{g^{-1}}(z)^{\frac{\nu}{p}} f\left(g^{-1} z\right),
$$

and it forms a projective representation of $G_{c}$. Let $R$ be the restriction map $R$ : $H^{\nu} \rightarrow C^{\infty}(D)$ by

$$
R f(x)=f(x) h(x)^{\frac{\nu}{2}} .
$$

Consider the restriction of the group action $\pi_{\nu}$ of $G_{c}$ to its subgroup $G$. Then $R$ is an $G$-intertwining map, as one can easily check from the transformation properties of $h(x, x)$. Consider its formal conjugate operator $R^{*}$ from $L^{2}\left(D, d \mu_{0}\right)$ to $H^{\nu}$ and form the operator $R^{*} R$ on $L^{2}(D, \omega)$. It is of the form, up to a constant,

$$
B_{\nu} f(z)=c_{\nu} \int_{D} f(w) \frac{h(z)^{\frac{\nu}{2}} h(w)^{\frac{\nu}{2}}}{h(z, w)^{\nu}} \frac{d m(w)}{h(w)^{\frac{p}{2}}},
$$

where the constant $c_{\nu}$ is a constant normalized so that $B_{\nu} 1=1$.

We note that the kernel $h(z, w)$ actually is positive, so there is no ambiguity about its power $h(z, w)^{\nu}$. Indeed by the transformation rule of $h(z, w)$ on $\mathbb{D} \times \mathbb{D}$

$$
h(g z, g w)=J_{g}(z)^{\frac{1}{p}} h(z, w) \overline{J_{g}(z)^{\frac{1}{p}}}
$$

for $g \in G_{c}$ and $(z, w) \in \mathbb{D} \times \mathbb{D}$, and that $h(0, w)=1$ we know that $h(z, w)$ is nonvanishing on $\mathbb{D} \times \mathbb{D}$. Moreover, $h(z, w)$ on $D \times D$ is a real-valued function. Thus being a continuous nonvanishing real-valued function on $D \times D$, it must be positive. Consequently, we can write $B_{\nu}$ as

$$
B_{\nu} f(z)=c_{\nu} \int_{D} \frac{h(z, z)^{\frac{\nu}{2}} h(w, w)^{\frac{\nu}{2}}}{\left|h(z, w)^{\frac{\nu}{2}}\right|^{2}} f(w) \frac{d m(w)}{h(w)^{\frac{p}{2}}} .
$$




\section{Siegel domain Realization of ReAl Bounded Symmetric Domains AND THE HARISH-CHANDRA $e_{\underline{\lambda}}$-FUNCTIONS}

The complex bounded symmetric domain $\mathbb{D}$ can be also realized as a Siegel domain in $V_{\mathbb{C}}$ via Cayley transform. The Cayley transform at the same time maps $D$ onto an unbounded domain in $V$, which will be its Siegel domain realization. The advantage with the unbounded realization is that the Harish-Chandra functions $e_{\underline{\lambda}}$ have explicit form.

Let $\left\{e_{1}, \cdots, e_{r}\right\}$ be a frame of $V$ and $e=e_{1}+\cdots+e_{r} ; e$ is then a maximal tripotent of $V$ and $V_{\mathbb{C}}$. Let

$$
V=V_{2} \oplus V_{1}
$$

be the Peirce decomposition of $V$ with respect to $e$, where

$$
V_{j}=\{z \in V ; D(e, e) z=2 z\}, \quad j=1,2 .
$$

The space $V_{2}$ is a Jordan algebra with the product $z \circ w=\frac{1}{2}\{z \bar{e} w\}$ and with unit $e$. Denote $x^{-1}$ the inverse of $x \in V_{2}$ with respect to this product. The Cayley transform is defined by

$$
\gamma_{e}(x)=\left(e+x_{2}\right) \circ\left(e-x_{2}\right)^{-1} \oplus \sqrt{2}\left\{\left(e-x_{2}\right)^{-1} \bar{e} x_{1}\right\} .
$$

To describe the image of $\gamma_{e}$ we consider the involution $Q(e): x \mapsto x^{*}=Q(e) \bar{x}$. Let $A$ and $B$ be the eigenspaces of the involution with eigenvalues 1 and -1 , respectively. The subspace $A$ is an Euclidean Jordan algebra. We will write $\Re(x)$ as the $A$-part of an element $x \in V_{2}$. Let $\Omega$ be the cone of positive elements in $A$. Denote $F\left(x_{1}, x_{1}\right)=D(e, \bar{e}) x_{1}$. It maps $V_{1}$ into $A$. The image of $D$ under $\gamma_{e}$ is the unbounded domain

$$
\mathcal{S}=\left\{x=x_{2} \oplus x_{1} \in V=V_{2} \oplus V_{1} ; x_{2}+x_{2}^{*}-F\left(x_{1}, x_{1}\right) \in \Omega\right\} .
$$

We observe that the differential of $\gamma_{e}$ at $z=0$ is

$$
\left(\begin{array}{cc}
2 & 0 \\
0 & \sqrt{2}
\end{array}\right)
$$

under the decomposition of $V=V_{2} \oplus V_{1}$.

We will denote the conjugation of the group $G$ also by $G$. Thus $\mathcal{S}=G / K$ with $e \in \mathcal{S}$ being the base point. We will hereafter fix this realization of $G / K$. Let $\mathfrak{g}=\mathfrak{k} \oplus \mathfrak{p}$ be the Cartan decomposition of the Lie algebra $\mathfrak{g}$ of $G$. The elements $D\left(e_{j}, e_{j}\right), j=1, \ldots, r$, span then a maximal subspace $\mathfrak{a}$ of $\mathfrak{p}$ of dimension $r$. Let $\gamma_{j} \in \mathfrak{a}^{*}$ be the linear functional on $\mathfrak{a}$ defined by

$$
\gamma_{j}\left(D\left(e_{k}, e_{k}\right)\right)=2 \delta_{j, k}
$$

where $\delta_{j, k}$ is the Kronecker symbol. Then the root system $\Sigma(\mathfrak{g}, \mathfrak{a})$ is of the following type:

$\left(A_{r}\right): \Sigma(\mathfrak{g}, \mathfrak{a})=\left\{ \pm \gamma_{j}, \frac{\gamma_{j} \pm \gamma_{k}}{2}\right\}$

$\left(B_{r}\right): \Sigma(\mathfrak{g}, \mathfrak{a})=\left\{ \pm \frac{\gamma_{j}}{2}, \frac{\gamma_{j} \pm \gamma_{k}}{2}\right\}$

$\left(C_{r}\right): \Sigma(\mathfrak{g}, \mathfrak{a})=\left\{ \pm \gamma_{j}, \frac{\gamma_{j} \pm \gamma_{k}}{2}\right\}$

$\left(B C_{r}\right): \Sigma(\mathfrak{g}, \mathfrak{a})=\left\{ \pm \gamma_{j}, \pm \frac{\gamma_{j}}{2}, \frac{\gamma_{j} \pm \gamma_{k}}{2}\right\} ;$

$\left(D_{r}\right): \Sigma(\mathfrak{g}, \mathfrak{a})=\left\{\frac{\gamma_{j} \pm \gamma_{k}}{2}\right\}$. 
Let us arrange an ordering of the roots so that

$$
\gamma_{1}<\gamma_{2}<\cdots<\gamma_{r}
$$

For type $C$ and $B C$ we further let $\gamma_{1}>0$. The root space decomposition can be explicitly done in terms of the Peirce decomposition of $V$. We present below formulas for the positive root spaces, which will be sufficient for our purposes. For the complex bounded symmetric domain $\mathbb{D}$ and its Siegel domain realization, the corresponding root spaces are calculated in [13] and [17].

Let

$$
V=\sum_{1 \leq j \leq k} V_{j k} \oplus \sum_{j=1}^{r} V_{0 j}
$$

be the joint Peirce decomposition with respect to $e_{1}, \cdots, e_{j}$, and

$$
V_{j k}=A_{j k} \oplus B_{j k}
$$

the Cartan decomposition of $V_{j k}, 1 \leq j \leq k$, under the involution $z \mapsto Q(e) \bar{z}$. With the notation in (2.1) we have

$$
V_{2}=\sum_{1 \leq j \leq k} V_{j k}, \quad V_{1}=\sum_{j=1}^{r} V_{0 j}
$$

and $V_{2}=A \oplus B$,

$$
A=\sum_{1 \leq j \leq k} A_{j k}, \quad B=\sum_{1 \leq j \leq k} B_{j k}
$$

We give the explicit formulas for the positive root spaces, in the bounded realization, it is essentially in 9], Propositions 11.18 and 9.19.

Proposition 2.1. The positive root spaces are given by

$$
\begin{gathered}
\mathfrak{g}_{\gamma_{j}}=B_{j, j}, \\
\mathfrak{g}_{\frac{\gamma_{j}}{2}}=\left\{v+D(e, \bar{v}) ; v \in V_{0, j}\right\}, \\
\mathfrak{g}_{\frac{\gamma_{j}-\gamma_{k}}{2}}=\left\{D\left(v, e_{k}\right) ; v \in A_{k j}\right\}, \quad j>k, \\
\mathfrak{g}_{\frac{\gamma_{j}+\gamma_{k}}{2}}=B_{k j}, \quad j>k .
\end{gathered}
$$

The type $D_{2}$ is somewhat special and will be treated separately in Section 5 . For all other types we define the invariants

$$
\iota=\operatorname{dim} V_{j j}, a=\operatorname{dim} A_{j k}, b=\operatorname{dim} V_{j 0},
$$

where $0<j<k$. They are independent of $j, k$ and the choice of the frame. Observe that $\operatorname{dim} A_{j j}=1$ and $\operatorname{dim} B_{j j}=\iota-1 ; \operatorname{dim} B_{j k}=\operatorname{dim} A_{j k}=a$ for type $B C, C$ and $D_{r}(r \geq 3)$, and $\operatorname{dim} B_{j k}=0$ for type $A$. Also $\iota=1$ for type $B_{r}$ and $D_{r}(r \geq 3)$. The dimensions of $A, B, V_{2}$ can then be calculated in terms of those invariants

$$
n_{A}=\operatorname{dim} A=r+\frac{a}{2} r(r-1), \quad n_{B}=\operatorname{dim} B=r(\iota-1)+\frac{a}{2} r(r-1),
$$

and $n_{2}=\operatorname{dim} V_{2}=n_{A}+n_{B}$. Recall that the genus $p$ of the complex bounded symmetric domain $D$ is defined

$$
p=p(\mathbb{D})=\frac{1}{r(\mathbb{D})}\left(2 n_{2}+n_{1}\right)
$$

where $r(\mathbb{D})$ is the rank of $\mathbb{D}$. 
Definition 2.2. The genus $p_{0}$ of $D$ is defined by

$$
p_{0}=p(D)=\frac{1}{2 r}\left(2 n_{2}+n_{1}\right) \text {. }
$$

Note that the genus $p_{0}=p(D)=\frac{1}{2} p(\mathbb{D})$ if it is of type $A, B$ or $D$, and $p(D)=$ $p(\mathbb{D})$ if it is of type $B C$ or $C$. (The rank of the complex domain $\mathbb{D}$ is $2 r$ if $D$ is of type $B C$ or $C$.)

For $\underline{\lambda} \in\left(\mathfrak{a}^{*}\right)^{\mathbb{C}}$ we write

$$
\underline{\lambda}=\sum_{j=1}^{r} \lambda_{j} \gamma_{j}
$$

and we will identify $\underline{\lambda}$ with its coordinates $\left(\lambda_{1}, \ldots, \lambda_{r}\right)$.

We let $\underline{\rho}=\sum_{j=1}^{r} \rho_{j} \gamma_{j}$ be the half sum of positive roots. Then

$$
\rho_{j}=\frac{1}{2}\left(\left(\iota-1+a(j-1)+\frac{b}{2}\right), \quad 1 \leq j \leq r,\right.
$$

for type $B, B C, C, D_{r}(r \geq 3)$, and

$$
\rho_{j}=\frac{a}{4}(2 j-(r+1))
$$

for type $A$.

Let $G=N A K$ be the corresponding Iwasawa decomposition of $G$. The HarishChandra $e_{\underline{\lambda}}$ on $\mathcal{S}=G / K$ is defined by

$$
e_{\underline{\lambda}}(z)=e^{(\underline{\lambda}+\rho) A(g)}
$$

where $g \in G$ is such that $z=g K$ and $A(g)$ is the a-part of $g \in G$ in its Iwasawa decomposition: $g=n \exp (A(g)) k$.

We will need an explicit formula for the function $e_{\underline{\lambda}}$. For that purpose we introduce the conical functions. Let $\Omega$ be the symmetric cone of positive elements in $A$. Then the Harish-Chandra $e$-function on $\Omega$ can be expressed in terms of the conical functions on $A$. So let $\delta(x)$ be the determinant function of the Jordan algebra $A$. Put $e^{(j)}=e_{1}+\cdots+e_{j}$ and consider the Peirce decomposition of $A$ with respect to $e^{(j)}, A=A_{2}\left(e^{(j)}\right) \oplus A_{1}\left(e^{(j)}\right) \oplus A_{0}\left(e^{(j)}\right)$. Let $\delta_{j}$ be the determinant function for the Jordan algebra $A_{2}\left(e^{(j)}\right)$ with identity $e^{(j)}$ and extend it to a polynomial function of $A$ via the projection from $A$ onto $A_{2}\left(e^{(j)}\right)$. For $\underline{\alpha}=\left(\alpha_{1}, \cdots, \alpha_{r}\right) \in \mathbb{C}^{r}$, we denote

$$
\delta_{\underline{\alpha}}(x)=\delta_{1}(x)^{\alpha_{1}-\alpha_{2}} \cdots \delta_{r-1}^{\alpha_{r-1}-\alpha_{r}}(x) \delta_{r}(x)^{\alpha_{r}}, \quad x \in \Omega .
$$

The $\delta_{\underline{\alpha}}$ is the Harish-Chandra $e$-function on $\Omega$. To find the $e$-function on $\mathcal{S}$ we let $\Delta$ be the determinant function of Jordan algebra $V_{2}$. On $\Omega \subset A \subset V_{2}, \Delta=\delta$ and has degree $r$ if $\Sigma(\mathfrak{g}, \mathfrak{a})$ is of type $A_{r}, B_{r}$ or $D_{r} ; \Delta=\delta^{2}$ and is of degree $2 r$ for type $B C_{r}$ and $C_{r}$; see e.g. [8]. We define similarly the functions $\Delta_{\underline{\alpha}}$ on $V_{2}$.

Lemma 2.3. The Harish-Chandra $e_{\underline{\lambda}}$-function on $\mathcal{S}$ is given by

$$
\begin{aligned}
e_{\underline{\lambda}}(z) & =\Delta_{\underline{\alpha}}\left(\Re\left(z_{2}\right)-\frac{1}{2} F\left(z_{1}, z_{1}\right)\right) \\
& =\Delta_{\underline{\alpha}}\left(\frac{1}{2}\left(z_{2}+z_{2}^{*}\right)-\frac{1}{2} F\left(z_{1}, z_{1}\right)\right), \quad z=z_{2} \oplus z_{1} \in \mathcal{S},
\end{aligned}
$$

with

$$
\underline{\alpha}=\underline{\lambda}+\underline{\rho}
$$


if $\Sigma(\mathfrak{g}, \mathfrak{a})$ is of type $A, B$, or $D$, and

$$
\underline{\alpha}=\frac{1}{2}(\underline{\lambda}+\underline{\rho})
$$

for type $B C$ or $C$.

Proof. Indeed the function $\Delta_{\underline{\alpha}}\left(\Re\left(z_{2}\right)-\frac{1}{2} F\left(z_{1}, z_{1}\right)\right)$ and $e_{\underline{\lambda}}$ coincides on $A \cdot e$ $=\exp (\mathfrak{a}) \cdot e$. To prove that they are the same we only need to know that $\Delta_{\underline{\alpha}}\left(\Re\left(z_{2}\right)-\frac{1}{2} F\left(z_{1}, z_{1}\right)\right)$ is invariant under $N$. By Proposition 3.1 it is clear that the function is invariant under the subgroups $\exp \left(\mathfrak{g}_{\gamma_{j}}\right)$ and $\exp \left(\mathfrak{g}_{\frac{\gamma_{j}+\gamma_{k}}{2}}\right)$, since $\Re\left(z_{2}\right)-\frac{1}{2} F\left(z_{1}, z_{1}\right)$ does not depend on the $B$-part of $z_{2}$. We consider now $\frac{\mathfrak{g}_{\gamma_{j}-\gamma_{k}}}{2}$, $j>k$. In view of Proposition 2.1, we see that the elements in the space, when considered as elements acting on $V=V_{2} \oplus V_{1}=A \oplus B \oplus V_{1}$, annihilate $V_{1}$ and keep the subspace $A$ invariant. When restricted to $A$, the elements in $\frac{\mathfrak{g}_{\frac{\gamma_{j}-\gamma_{k}}{2}}}{2}$ are in the Lie algebra of the corresponding Iwasawa $N$-group of the symmetric cone $\Omega$ and thus keep the conical function $\Delta_{\alpha}(x)$ invariant (see [3], Chapter XI. Section 2). Consequently, $\Delta_{\underline{\alpha}}\left(\Re\left(z_{2}\right)-\frac{1}{2} F\left(z_{1}, z_{1}\right)\right)$ is invariant under $\exp \left(\mathfrak{g}_{\frac{\gamma_{j}-\gamma_{k}}{2}}\right)$. Finally, consider an element $v+D(e, \bar{v}) \in \mathfrak{g}_{\frac{\gamma_{j}}{2}}, v \in V_{0 j}$. It follows from [9], Lemma 10.7 (and its proof) that

$$
\exp (v+D(e, \bar{v}))=\exp \left(v+\frac{1}{2} F(v, v)\right) \exp (D(e, \bar{v}))
$$

where the element $\exp \left(v+\frac{1}{2} F(v, v)\right)$ acts on vectors in $V$ via translation, and that

$$
\exp (D(e, \bar{v}))\left(z_{2} \oplus z_{1}\right)=\left(z_{2}+F\left(z_{1}, v\right)\right) \oplus z_{1}
$$

Thus

$$
\exp (v+D(e, \bar{v}))\left(z_{2} \oplus z_{1}\right)=\left(z_{2}+F\left(z_{1}, v\right)+\frac{1}{2} F(v, v)\right) \oplus\left(z_{1}+v\right) .
$$

From this formula we see that the action $\exp (v+D(e, \bar{v}))$ on $\left(z_{2} \oplus z_{1}\right)$ keeps $\Re\left(z_{2}\right)-$ $\frac{1}{2} F\left(z_{1}, z_{1}\right)$, and consequently $\Delta_{\underline{\alpha}}\left(\Re\left(z_{2}\right)-\frac{1}{2} F\left(z_{1}, z_{1}\right)\right)$, invariant. This completes the proof.

The Berezin transform on $\mathcal{S}$ is then of the form

$$
B_{\nu} f(z)=C_{\nu} \int_{\mathcal{S}} f(w) \frac{\Delta\left(\Re z_{2}-\frac{1}{2} F\left(z_{1}, z_{1}\right)\right)^{\frac{\nu}{2}} \Delta\left(\Re w_{2}-\frac{1}{2} F\left(w_{1}, w_{1}\right)\right)^{\frac{\nu}{2}}}{\left(\Delta\left(\frac{1}{2}\left(z_{2}+w_{2}^{*}\right)-\frac{1}{2} F\left(z_{1}, w_{1}\right)\right)\right)^{\nu}} d \mu_{0}(w)
$$

where

$$
d \mu_{0}(w)=\frac{d m(w)}{\Delta\left(\Re w_{2}-\frac{1}{2} F\left(w_{1}, w_{1}\right)\right)^{\frac{p}{2}}},
$$

is the $G$-invariant measure on $\mathcal{S}$. Here $C_{\nu}$ is a constant normalized so that $B_{\nu} 1=1$, its exact value will be given in the following sections.

As will be proved in Section 7, the operator $B_{\nu}$ is a positive bounded operator on $L^{2}\left(\mathcal{S}, d \mu_{0}\right)$ when $\nu$ is in a certain interval. Then the Harish-Chandra $e$-function $e_{\underline{\lambda}}$ is an eigenfunction of $B_{\nu}$ with eigenvalue $b_{\nu}(\underline{\lambda})$, in the sense of spectral calculus. Namely,

$$
B_{\nu} e_{\underline{\lambda}}(z)=b_{\nu}(\underline{\lambda}) e_{\underline{\lambda}}(z)
$$


Taking $z=e$ as the base point we get formally $b_{\nu}(\underline{\lambda})=B_{\nu} e_{\underline{\lambda}}(e)$. Denote

$$
I(\nu, \underline{\alpha})=\int_{\mathcal{S}} e_{\underline{\lambda}}(w) \frac{\Delta\left(\Re w_{2}-\frac{1}{2} F\left(w_{1}, w_{1}\right)\right)^{\frac{\nu}{2}}}{\left(\Delta\left(\frac{1}{2}\left(e+w_{2}^{*}\right)\right)\right)^{\nu}} d \mu_{0}(w) .
$$

Here $\underline{\alpha}$ and $\underline{\lambda}$ are related as in (2.7) and (2.8). Then $b_{\nu}(\underline{\lambda})=C_{\nu} I(\nu, \underline{\alpha})$. So our main goal is the calculation of the integral $I(\nu, \underline{\alpha})$. Note that the normalization constant $C_{\nu}=I(\nu, 0)^{-1}$, and will be found at the same time.

We will prove that the integral is convergent for $\underline{\lambda}$ in a certain domain in $\left(\mathfrak{a}^{*}\right)^{\mathbb{C}}$. However, the function $e_{\underline{\lambda}}$ is $N$-invariant and transforms under $A$ by the character $e^{\underline{\lambda}}$ and $N A$ acts transitively on $\mathcal{S}$. Thus, if the integral (2.10) is absolutely convergent, that is, if the integral defining the Berezin transform $B_{\nu} e_{\underline{\lambda}}(z)$ at $z=e$ is absolutely convergent, then by a simple change of variable argument we know that the integral $B_{\nu} e_{\underline{\lambda}}(z)$ is absolutely convergent for all $z \in \mathcal{S}$; see [1].

We recall finally the Gindikin Gamma function on a symmetric cone; see e.g. [3], Chapter VII. Consider the Laplace transform of the function $\delta_{\underline{\alpha}}(x) \delta(x)^{-\frac{n_{A}}{r}}$,

$$
\int_{\Omega} e^{-(u, x)} \delta_{\underline{\alpha}}(x) \delta(x)^{-\frac{n_{A}}{r}} d x,
$$

for $u \in \Omega$. The integral is convergent if and only if $\Re\left(\alpha_{j}\right)>(j-1) a / 2$ for $j=1,2, \cdots, r$. Moreover,

$$
\int_{\Omega} e^{-(u, x)} \delta_{\underline{\alpha}}(u) \delta(x)^{-\frac{n_{A}}{r}} d x=\Gamma_{\Omega}(\underline{\alpha}) \delta_{\underline{\alpha}^{*}}^{*}(u),
$$

where

$$
\Gamma_{\Omega}(\underline{\alpha})=(2 \pi)^{\left(n_{A}-r\right) / 2} \prod_{j=1}^{r} \Gamma\left(\alpha_{j}-(j-1) a / 2\right),
$$

and $\underline{\alpha}^{*}=\left(-\alpha_{r}, \ldots,-\alpha_{1}\right)$ and $\delta_{\beta}^{*}(x)$ for $\underline{\beta}=\left(\beta_{1}, \ldots, \beta_{r}\right)$ is the conical function defined via Peirce decomposition with respect to $e_{r}, e_{r}+e_{r-1}, \ldots, e_{r}+e_{r-1}+\cdots+e_{1}$.

\section{Calculations of the symbol: Type BC and C}

The type $\mathrm{C}$ root systems can be viewed as special cases of the type $\mathrm{BC}$ with the root multiplicity of $\frac{\gamma_{j}}{2}$ being 0 (and the other types can be viewed as degenerate cases). So we consider the type BC. Notice that the genus $p_{0}=\iota+a(r-1)+\frac{b}{2}$.

The determinant function $\Delta$ of $V_{2}$ when restricted to the Jordan subalgebra $A$ is the square of the determinant function of that of $A$. To distinguish them we write as before $\delta(z)$ the determinant function of $A$. Thus

$$
\Delta(x)=\delta(x)^{2}, \quad x \in A .
$$

We present first some integral formulas.

Proposition 3.1. Suppose $\nu>\iota-2+\frac{1}{2}(1+a(r-1))$. The following integral formula

$$
I_{B}(\nu)=\int_{B} \frac{d \eta}{\Delta(e-\eta)^{\nu}}=4^{n_{A}-r(\nu-(\iota-2))} \sqrt{\pi}^{r(\iota-2)+2 n_{A}} \frac{\Gamma_{\Omega}\left(2 \nu-2(\iota-2)-\frac{n_{A}}{r}\right)}{\Gamma_{\Omega}(\nu) \Gamma_{\Omega}\left(\nu-\frac{\iota-2}{2}\right)}
$$

holds and the integral is absolutely convergent. 
Notice that

$$
\begin{aligned}
\Delta(e-\eta)^{2} & =\Delta(e-\eta) \Delta(e+\eta)=\Delta\left(e-\eta^{2}\right) \\
& =\Delta\left(e+\eta \circ \eta^{*}\right)=\delta\left(e+\eta \circ \eta^{*}\right)^{2} .
\end{aligned}
$$

The integral $I_{B}(\nu)$ is now

$$
I_{B}(\nu)=\int_{B} \frac{d \eta}{\delta\left(e+\eta \circ \eta^{*}\right)^{\nu}}
$$

To prove the integral formula we need the following result; see 3], Chapter VII, Ex. 5.

Lemma 3.2. Suppose $\beta>\frac{1}{2}(1+a(r-1))$. The integral formula

$$
I_{A}(\beta)=\int_{A} \frac{d t}{|\delta(e+i t)|^{2 \beta}}=4^{n_{A}-r \beta} \pi^{n_{A}} \frac{\Gamma_{\Omega}\left(2 \beta-\frac{n_{A}}{r}\right)}{\Gamma_{\Omega}(\beta)^{2}}
$$

holds and the integral is absolutely convergent.

The integrals $I_{A}(\beta)$ and $I_{B}(\nu)$ above can also be written as an integration on the symmetric cone $\Omega$ in $A$; by comparing the two formulas we will then be able to calculate $I_{B}(\nu)$.

Indeed, we write $|\delta(e+i t)|^{2}=\delta(e+i t) \overline{\delta(e+i t)}=\delta(e+i t) \delta(e-i t)=\delta\left(e+t^{2}\right)$, and use the Gindikin Gamma function formula (2.11)

$$
\begin{gathered}
\delta\left(e+t^{2}\right)^{-\beta}=\frac{1}{\Gamma_{\Omega}(\beta)} \int_{\Omega} e^{-\left(e+t^{2}, u\right)} \delta(u)^{\beta-\frac{n_{A}}{r}} d u \\
I_{A}(\beta)=\int_{A} \frac{d t}{|\delta(e+i t)|^{2 \beta}}=\frac{1}{\Gamma_{\Omega}(\beta)} \int_{A} \int_{\Omega} e^{-\left(e+t^{2}, u\right)} \delta(u)^{\beta-\frac{n_{A}}{r}} d u d t \\
=\frac{1}{\Gamma_{\Omega}(\beta)} \int_{\Omega} \int_{A} e^{-(e, u)} e^{-\left(t^{2}, u\right)} \delta(u)^{\beta-\frac{n_{A}}{r}} d t d u .
\end{gathered}
$$

Namely,

$$
I_{A}(\beta)=\frac{1}{\Gamma_{\Omega}(\beta)} \int_{\Omega} G_{A}(u) e^{-(e, u)} \delta(u)^{\beta-\frac{n_{A}}{r}} d t d u
$$

with

$$
G_{A}(u)=\int_{A} e^{-\left(t^{2}, u\right)} d t, \quad u \in \Omega,
$$

being a Gaussian type integral. Let $u=\sum_{j=1}^{r} u_{j} c_{j}$ be the Peirce decomposition of $u$ with $c_{j}$ being minimal orthogonal tripotents, and $u_{j}>0$ since $u \in \Omega$. Let $A=\sum A_{j k}$ be the joint Peirce decomposition and write $t=\sum t_{j k}$. Then we find that, by the Peirce rule (see [9], Theorem 3.15, Corollary 3.16)

$$
\left(t^{2}, u\right)=\sum_{j<k} \frac{u_{j}+u_{k}}{2}\left(t_{j k}, t_{j k}\right)+\sum_{j} u_{j}\left(t_{j j}, t_{j j}\right) .
$$

Thus

$$
\begin{aligned}
G_{A}(u) & =\prod_{j=1}^{r} \int_{A_{j j}} e^{-u_{j}(t, t)} \prod_{j<k} \int_{A_{j k}} e^{-\frac{1}{2}\left(u_{j}+u_{k}\right)(t, t)} d t \\
& =\sqrt{\pi}^{r+\frac{a}{2} r(r-1)}\left(\prod_{j=1}^{r} u_{j}\right)^{-1}\left(\prod_{j<k} \frac{u_{j}+u_{k}}{2}\right)^{-a} .
\end{aligned}
$$


Next we prove Proposition 3.1.

Proof. Using (3.2) and performing the same calculation as above (with $A$ replaced by $B$ ) we get

$$
I_{B}(\nu)=\frac{1}{\Gamma_{\Omega}(\nu)} \int_{\Omega} G_{B}(u) e^{-(e, u)} \delta(u)^{\nu-\frac{n_{A}}{r}} d t d u
$$

with

$$
G_{B}(u)=\int_{B} e^{\left(t^{2}, u\right)} d t=\int_{B} e^{-\left(t \circ t^{*}, u\right)} d t
$$

Consider as above the Peirce decomposition of $u$ and $B$. We can then calculate $G_{B}(u)$ and obtain

$$
G_{B}(u)=\sqrt{\pi}^{r(\iota-1)+\frac{a}{2} r(r-1)}\left(\prod_{j=1}^{r} u_{j}\right)^{-(\iota-1)}\left(\prod_{j<k} \frac{u_{j}+u_{k}}{2}\right)^{-a} .
$$

Therefore, comparing $G_{B}(u)$ with $G_{A}(u)$ given in (3.5) it follows that

$$
G_{B}(u)=\sqrt{\pi}^{r(\iota-2)}\left(\prod_{j=1}^{r} u_{j}\right)^{-(\iota-2)} G_{A}(u)=\sqrt{\pi}^{r(\iota-2)} \delta(u)^{-(\iota-2)} G_{A}(u) .
$$

Substituting this into (3.6) we find

$$
I_{B}(\nu)=\sqrt{\pi}^{r(\iota-2)} \frac{1}{\Gamma_{\Omega}(\nu)} \int_{\Omega} e^{-(e, u)} \delta(u)^{\nu-\frac{n_{A}}{r}} \delta(u)^{-(\iota-2)} G_{A}(u) d u .
$$

Comparing this with (3.4) we find that the integral $I_{B}(\nu)$ is of the same type as $I_{A}(\beta)$ with $\beta=\nu-(\iota-2)$; more precisely,

$$
I_{B}(\nu)=\sqrt{\pi}^{r(\iota-2)} \frac{\Gamma_{\Omega}(\nu-(\iota-2))}{\Gamma_{\Omega}(\nu)} I_{A}(\nu-(\iota-2)) .
$$

which again by Lemma 3.2 is

$$
I_{B}(\nu)=4^{n_{A}-r(\nu-(\iota-2))} \sqrt{\pi}^{r(\iota-2)+2 n_{A}} \frac{\Gamma_{\Omega}\left(2 \nu-2(\iota-2)-\frac{n_{A}}{r}\right)}{\Gamma_{\Omega}(\nu) \Gamma_{\Omega}\left(\nu-\frac{\iota-2}{2}\right)} .
$$

We can now calculate the integral $I(\nu, \underline{\alpha})$.

Proposition 3.3. Suppose $\nu>p_{0}-1=p-1$. The integral $I(\nu, \underline{\alpha})$ is absolutely convergent if

$$
-\frac{1}{2}\left(\left(\nu-p_{0}+1\right)+\frac{a}{2}(r-j)\right)<\alpha_{j}<\frac{1}{2}\left(\nu-\frac{a}{2}(r-j)\right), \quad 1 \leq j \leq r,
$$

and its value is given by

$$
I(\nu, \underline{\alpha})=2^{2 r \nu} \sqrt{2 \pi}^{r b} I_{B}(\nu) \frac{\Gamma_{\Omega}\left(\nu-p_{0}+\frac{n_{A}}{r}+2 \underline{\alpha}\right) \Gamma_{\Omega}\left(\nu+p_{0}+1+\frac{n_{2}}{r}-\frac{b}{2}+2 \underline{\alpha}^{*}\right)}{\Gamma_{\Omega}\left(2 \nu-\frac{n_{B}}{r}\right)} .
$$

Proof. We write $w=w_{2}+w_{1}$ and $w_{2}=x+y$ according to the Peirce decomposition of $V=V_{2} \oplus V_{1}$ and the Cartan decomposition $V_{2}=A \oplus B$. We perform the change of variables $\xi=x-\frac{1}{2} F\left(w_{1}, w_{1}\right)$. Thus $\mathcal{S}$ is parametrized by the product 
$\Omega \times B \times V_{1}$. In terms of the new coordinates, $w_{2}=x+y=\xi+\frac{1}{2} F\left(w_{1}, w_{1}\right)+y$ and $w_{2}^{*}=\xi+\frac{1}{2} F\left(w_{1}, w_{1}\right)-y$. The integral can be rewritten as

$$
2^{2 r \nu} \int_{\Omega \times V_{1} \times B} \Delta_{\underline{\alpha}}(\xi) \frac{\Delta(\xi)^{\frac{\nu}{2}-\frac{p}{2}}}{\Delta\left(e+\xi+\frac{1}{2} F\left(w_{1}, w_{1}\right)-y\right)^{\nu}} d y d w_{1} d \xi .
$$

(Here the constant $2^{2 r \nu}$ appears because $\Delta$ is of degree $2 r$, and $\Delta\left(\frac{e+w_{2}^{*}}{2}\right)=$ $2^{-2 r} \Delta\left(e+w_{2}^{*}\right)$.) We consider first the integral with respect to $y$. Denote temporarily $u=e+\xi+\frac{1}{2} F\left(w_{1}, w_{1}\right) \in \Omega$ and note that

$$
\begin{aligned}
& \Delta\left(e+\xi+\frac{1}{2} F\left(w_{1}, w_{1}\right)-y\right) \\
& \quad=\Delta(u-y)=\Delta\left(P\left(u^{\frac{1}{2}}\right) e-P\left(u^{\frac{1}{2}}\right)\left(P\left(u^{-\frac{1}{2}}\right)(y)\right)\right. \\
& \quad=\Delta(u) \Delta\left(e-P\left(u^{-\frac{1}{2}}\right)(y)\right)=\delta(u)^{2} \Delta\left(e-P\left(u^{-\frac{1}{2}}\right)(y)\right) .
\end{aligned}
$$

Change variables $\eta=P\left(u^{-\frac{1}{2}}\right)(y)$. Then $d m(\eta)=\delta(u)^{-\frac{n_{2}-n_{A}}{r}} d y$ since $\operatorname{dim} B=$ $n_{2}-n_{A}$. The integration with respect to $y$ becomes,

$$
\delta(u)^{\frac{n_{2}-n_{A}}{r}-2 \nu} \int_{B} \Delta(e-\eta)^{-\nu} d \eta=\delta(u)^{\frac{n_{2}-n_{A}}{r}-2 \nu} I_{B}(\nu)
$$

and $I_{B}(\nu)$ is given by Proposition 3.1.

We consider now the integral with respect to $w_{1}$, which appears in $u$. We have

$$
\delta(u)^{\frac{n_{2}-n_{A}}{r}-2 \nu}=\frac{1}{\Gamma\left(2 \nu-\frac{n_{2}-n_{A}}{r}\right)} \int_{\Omega} e^{-(u, \zeta)} \delta(\zeta)^{2 \nu-\frac{n_{2}}{r}} d \zeta
$$

and $(u, \zeta)=(e+\xi, \zeta)+\frac{1}{2}\left(F\left(w_{1}, w_{1}\right), \zeta\right)$. Thus

$$
\begin{aligned}
& \int_{V_{1}} \delta(u)^{\frac{n_{2}-n_{A}}{r}-2 \nu} d w_{1} \\
= & \frac{1}{\Gamma\left(2 \nu-\frac{n_{2}-n_{A}}{r}\right)} \int_{V_{1}} \int_{\Omega} e^{-(e+\xi, \zeta)} e^{-\frac{1}{2}\left(F\left(w_{1}, w_{1}\right), \zeta\right)} \delta(\zeta)^{2 \nu-\frac{n_{2}}{r}} d \zeta d w_{1} \\
= & \frac{1}{\Gamma\left(2 \nu-\frac{n_{2}-n_{A}}{r}\right)} \int_{\Omega} e^{-(e+\xi, \zeta)} \delta(\zeta)^{2 \nu-\frac{n_{2}-n_{A}}{r}}\left(\int_{V_{1}} e^{-\frac{1}{2}\left(F\left(w_{1}, w_{1}\right), \zeta\right)} d w_{1}\right) d \zeta,
\end{aligned}
$$

and the change of the order of integration will be justified by our method calculation. Writing

$$
\begin{aligned}
\left(F\left(w_{1}, w_{1}\right), \zeta\right) & =\left(F\left(w_{1}, w_{1}\right), P\left(\zeta^{\frac{1}{2}}\right) e\right)=\left(P\left(\zeta^{\frac{1}{2}}\right) F\left(w_{1}, w_{1}\right), e\right) \\
& =\left(F\left(\zeta^{\frac{1}{2}}\right) \circ w_{1}, \zeta^{\frac{1}{2}} \circ w_{1}, e\right)
\end{aligned}
$$

by [9], Proposition 10.11. Here $\zeta^{\frac{1}{2}} \circ w_{1}=\frac{1}{2} D\left(\zeta^{\frac{1}{2}}, e\right) w_{1}$ is the Jordan product on $V$. Performing a change of variables $v=\zeta^{\frac{1}{2}} \circ w_{1}$, we have $d v=\delta(\zeta)^{\frac{b}{2}} d w_{1}$ and

$$
\int_{V_{1}} e^{-\frac{1}{2}\left(F\left(w_{1}, w_{1}\right), \zeta\right)} d w_{1}=\delta(\zeta)^{-\frac{b}{2}} \int_{V_{1}} e^{-\frac{1}{2}(F(v, v), e)} d v ;
$$

the last integral is of Gaussian type and can be easily evaluated, observing

$$
\frac{1}{2}(F(v, v), e)=\frac{1}{2}(D(e, v) v, e)=\frac{1}{2}(v, D(v, e) e)=\frac{1}{2}(v, D(e, e) v)=\frac{1}{2}(v, v),
$$

so that

$$
\int_{V_{1}} e^{-\frac{1}{2}(F(v, v), e)} d v=\sqrt{2 \pi}^{r b}
$$


Continuing the formula (3.9), we find it is, disregarding the Gamma factor,

$$
\sqrt{2 \pi}^{r b} \int_{\Omega} e^{-(e+\xi, \zeta)} \delta(\zeta)^{2 \nu-\frac{n_{2}}{r}-\frac{b}{2}} d \zeta
$$

which is integrable if

$$
2 \nu-\frac{n_{2}}{r}-\frac{b}{2}>\frac{a}{2}(r-1),
$$

namely, if $\nu>\frac{1}{2}\left(\frac{n_{2}}{r}+\frac{b}{2}+\frac{a}{2}(r-1)\right)$ which is satisfied by our assumption of $\nu$. This consequently justifies the change of order of integration in (3.9).

Our integral in question is

$$
\begin{aligned}
& 2^{r \nu} \sqrt{2 \pi}^{r b} \frac{1}{\Gamma\left(2 \nu-\frac{n_{2}-n_{A}}{r}\right)} I_{B}(\nu) \\
& \quad \times \int_{\Omega} \int_{\Omega} e^{-(e, \zeta)} e^{-(\xi, \zeta)} \Delta_{\underline{\alpha}}(\xi) \Delta(\xi)^{\frac{\nu}{2}-\frac{p}{2}} \delta(\zeta)^{2 \nu-\frac{n_{2}-n_{A}}{r}-\frac{b}{2}} d \xi d \zeta .
\end{aligned}
$$

We calculate the integral with respect to $\xi$. Recall the formula (2.11) and that $\Delta(\xi)=\delta(\xi)^{2}$,

$$
\int_{\Omega} e^{-(\xi, \zeta)} \Delta_{\underline{\alpha}}(\xi) \Delta(\xi)^{\frac{\nu}{2}-\frac{p}{2}} d \xi=\Gamma_{\Omega}\left(2 \underline{\alpha}+\nu-p+\frac{n_{A}}{r}\right) \delta_{2 \underline{\alpha}^{*}-\nu+p-\frac{n_{A}}{r}}^{*}(\zeta),
$$

which is absolutely convergent if

$$
2 \underline{\alpha}_{j}+\nu-p+\frac{n_{A}}{r}>\frac{a}{2}(j-1), \quad j=1, \ldots, r .
$$

The remaining integral, disregarding the constant, is then

$$
\int_{\Omega} e^{-(e, \zeta)} \delta_{2 \underline{\alpha}^{*}-\nu+p-\frac{n_{A}}{r}}^{*}(\zeta) \delta(\zeta)^{2 \nu-\frac{n_{2}}{r}-\frac{b}{2}} d \zeta=\Gamma_{\Omega}\left(2 \underline{\alpha}^{*}+\nu+p-\frac{n_{2}}{r}-\frac{b}{2}\right) .
$$

But since $p=\frac{n_{2}}{r}+\frac{b}{2}$, the above is $\Gamma_{\Omega}\left(2 \underline{\alpha}^{*}+\nu\right)$. Moreover, the above integral is absolutely convergent if

$$
2\left(\underline{\alpha}^{*}\right)_{j}-\nu<\frac{a}{2}(j-1), \quad j=1, \ldots, r .
$$

The inequalities (3.10) and (3.11) combined give our condition $\underline{\alpha}$. This finishes the proof.

\section{Calculations of the symbol: Types $B_{r}(r \geq 1)$ and $D_{r}(r \geq 3)$}

In this section we calculate the integral $I(\nu, \underline{\alpha})$ for type $B_{r}(r \geq 1)$ and $D_{r}$ $(r \geq 3)$. The type $D_{r}(r \geq 3)$ can be viewed as a degenerate case of type $B_{r}$ with the root multiplicity of $\frac{\gamma_{j}}{2}$ being 0 . The method of calculation is basically the same as in the previous section. So we will only indicate the necessary changes needed in the calculations.

Proposition 3.1 in this case takes the following form; we remark that, as we did for the function $h(z, w)$ in Section 2, the function $\Delta(e-\eta)$ is positive on $B$.

Proposition 4.1. Suppose $\nu>a(r-1)-1$. The integral

$$
I_{B}(\nu)=\int_{B} \frac{d \eta}{\Delta(e-\eta)^{\nu}}
$$

is absolutely convergent and its values are given by

$$
I_{B}(\nu)=\sqrt{\pi}^{n_{2}} 4^{n_{A}-r\left(\frac{\nu}{2}+\frac{1}{2}\right)} \frac{\Gamma_{\Omega}\left(\nu+1-\frac{n_{A}}{r}\right)}{\Gamma_{\Omega}\left(\frac{\nu}{2}\right) \Gamma_{\Omega}\left(\frac{\nu}{2}+\frac{1}{2}\right)} .
$$


The value of the integral $I(\nu, \underline{\alpha})$ is given in the following; we note that in comparison with Types $\mathrm{BC}$ and $\mathrm{C}$, the convergence region of $\nu$ improves to $\nu>p-2$. (The discrete series are for $\nu>p-1$.)

Proposition 4.2. Suppose $\nu>2 p_{0}-2=p-2$, that is $\frac{\nu}{2}>p_{0}-1=\frac{p}{2}-1$. The integral $I(\nu, \underline{\alpha})$ is absolutely convergent if

$$
-\frac{1}{2}\left(\left(\nu-2 p_{0}+2\right)+a(r-j)\right)<\alpha_{j}<\frac{1}{2}(\nu-a(r-j)), \quad 1 \leq j \leq r .
$$

and its values are given by

$$
I_{\mathcal{S}}(\nu, \underline{\alpha})=\sqrt{2 \pi}^{r b} I_{B}(\nu) \frac{\Gamma_{\Omega}\left(\underline{\alpha}+\frac{\nu}{2}-\frac{p}{2}+\frac{n_{A}}{r}\right) \Gamma_{\Omega}\left(\underline{\alpha}^{*}+\frac{\nu}{2}\right)}{\Gamma_{\Omega}\left(\nu-\frac{n_{B}}{r}\right)} .
$$

Example 4.3. We consider the simple case of the unit disk $D$ in $\mathbb{R}^{2}$, considered as a real bounded symmetric domain in the unit ball $\mathbb{D}$ of $\mathbb{C}^{2}$. It is of type $B_{1}$ with roots $\pm \frac{\gamma}{2}$. The ball $\mathbb{D}$ is a realization of the Hermitian symmetric space $S U(2,1) / S(U(2) \times U(1))$, and the real disk of $S O(2,1) / S O(2)$. The genus $p$ of $\mathbb{D}$ is now 3 and the weighted Bergman space $H^{\nu}$ considered in Section 1 now has reproducing kernel $(1-\langle z, w\rangle)^{-\nu}$ with $\nu>p-1=2$. The Berezin transform on $D$ is now

$$
B_{\nu} f(y)=\frac{\nu-1}{2 \pi} \int_{D} f(x) \frac{\left(1-\|x\|^{2}\right)^{\frac{\nu}{2}}\left(1-\|y\|^{2}\right)^{\frac{\nu}{2}}}{(1-(x, y))^{\nu}} \frac{d x}{\left(1-\|x\|^{2}\right)^{\frac{3}{2}}},
$$

where $x=\left(x_{1}, x_{2}\right)$ and $(x, y)=x_{1} y_{1}+x_{2} y_{2}$. However, the unit disk $D=S O(2,1) /$ $S O(2)$ in $\mathbb{R}^{2}$ itself has a Hermitian structure defined by the Lie algebra of $S O(2)$, and in this realization it is not the standard Hermitian structure on $\mathbb{R}^{2}$ defined by $(u, v) \mapsto(-v, u)$ and $\frac{d u^{2}+d v^{2}}{\left(1-u^{2}-v^{2}\right)^{2}}$. However, those two structures are equivalent; denoting the unit disk in $\mathbb{C}=\mathbb{R}^{2}$ with the standard Hermitian structure by $D_{\mathbb{C}}$, a biholomorphic mapping from $D_{\mathbb{C}}$ onto $D=S O(2,1) / S O(2)$ is given by the Hua transformation

$$
z=u+i v \mapsto \frac{2}{1+|z|^{2}}(u, v) .
$$

See 4], Chapter X, Exercise D2. The corresponding Berezin transform on $D_{\mathbb{C}}$, obtained from (4.1) by conjugating the transformation, is then

$$
B_{\nu} f(w)=\frac{2(\nu-1)}{\pi} \int_{D_{\mathbb{C}}} f(z) \frac{\left(1-|z|^{2}\right)^{\nu}\left(1-|w|^{2}\right)^{\nu}}{\left|(1-z \bar{w})^{\nu}\right|^{2}}\left(1+\left|\frac{z-w}{1-z \bar{w}}\right|^{2}\right)^{-\nu} \frac{d m(z)}{\left(1-|z|^{2}\right)^{2}} .
$$

The symbol of $B_{\nu}$ in this case is, with $\lambda \gamma$ identified with $\lambda$

$$
b_{\nu}(\lambda)=\frac{\Gamma\left(\frac{\nu}{2}-\frac{1}{4}+\lambda\right) \Gamma\left(\frac{\nu}{2}-\frac{1}{4}-\lambda\right)}{\Gamma\left(\frac{\nu}{2}\right) \Gamma\left(\frac{\nu}{2}-\frac{1}{2}\right)} .
$$

It is interesting to see that the Berezin transform so obtained is quite close to the classical Berezin transform (0.1), whose symbol, after normalization, is

$$
\frac{\Gamma\left(\nu-\frac{1}{2}+\frac{\lambda}{2}\right) \Gamma\left(\nu-\frac{1}{2}-\frac{\lambda}{2}\right)}{\Gamma(\nu) \Gamma(\nu-1)} ;
$$

see [17] and [15]. 


\section{Calculations of the symbol: Type $A$ and $D_{2}$}

The integral $I(\nu, \underline{\alpha})$ for Type A is very simple. The real Siegel domain $\mathcal{S}=\Omega$, and our integral is

$$
2^{r \nu} \int_{\mathcal{S}} \Delta_{\alpha}(w) \frac{\Delta(w)^{\frac{\nu}{2}}}{\Delta(e+w)^{\nu}} \frac{d w}{\Delta(w)^{\frac{p}{2}}}
$$

which is a Beta-type integral. The result is as follows.

Proposition 5.1. Suppose $\nu>p-2=2 p_{0}-2$. The integral $I(\nu, \underline{\alpha})$ is absolutely convergent if

$$
\frac{1}{2}(\nu-a(j-1))<\alpha_{j}<\frac{1}{2}(\nu-a(r-j)), \quad 1 \leq j \leq r,
$$

and its value is given by

$$
2^{r \nu} \frac{\Gamma_{\Omega}\left(\frac{\nu}{2}+\underline{\alpha}\right) \Gamma_{\Omega}\left(\frac{\nu}{2}+\underline{\alpha}^{*}\right)}{\Gamma_{\Omega}(\nu)} .
$$

We now consider type $D_{2}$, the Jordan triple is denoted by $I V_{n}^{\mathbb{R}, m}\left(2 \leq m \leq\left[\frac{n}{2}\right]\right)$ in [9]. So we introduce the necessary notation. Let $V=\mathbb{R}^{n}$, and consider the quadratic operator $Q(x)$ on $V$ defined by

$$
Q(x) y=q(x, y) x-q(x) y
$$

where $q(x)=x_{1}^{2}+\cdots+x_{m}^{2}-x_{m+1}^{2}-\cdots-x_{n}^{2}$ and $q(x, y)$ is its polarization $q(x, y)=$ $q(x+y)-q(x)-q(y)$. The involution $\bar{x}$ of $x$ is $\bar{x}=\left(x_{1}, \cdots, x_{m},-x_{m+1}, \cdots,-x_{n}\right)$. Let $e_{1}=\frac{1}{2}(1,0, \cdots, 0,1)$ and $e_{2}=\frac{1}{2}(1,0, \cdots, 0, \ldots,-1)$ and $e=e_{1} \oplus e_{2}$. Then $e_{1}$ and $e_{2}$ form a frame of tripotents and $e$ is a maximal tripotent. The joint Peirce decomposition of $V$ is $V=V_{11} \oplus V_{22} \oplus V_{12}$ with $V_{j j}=\mathbb{R} e_{j}, j=1,2$. The Cartan involution $x \mapsto Q(e) \bar{x}$ gives a decomposition of $V, V=A \oplus B$ with $A=V_{11} \oplus V_{22} \oplus A_{12}$,

$$
A_{12}=\left\{x \in V ; x_{n}=x_{1}=x_{2}=\cdots=x_{m}=0\right\}
$$

and

$$
B=B_{12}=\left\{x \in V ; x_{1}=x_{m+1}=\cdots=x_{n}=0\right\},
$$

$\operatorname{dim} A_{12}=n-m-1, \operatorname{dim} B_{12}=m-1$. The determinant function $\Delta(x)=$ $x_{1}^{2}+\cdots+x_{n}^{2}$. In terms of the notation in Section 2, the positive roots are $\frac{\gamma_{2}+\gamma_{1}}{2}$, $\frac{\gamma_{2}-\gamma_{1}}{2}$ with root multiplicities $m-1$ and $n-m-1$ respectively, and

$$
\underline{\rho}=\frac{1}{4}\left((2 m-n) \gamma_{1}+(n-2) \gamma_{2}\right) .
$$

The symmetric cone in $A$ consists of all elements $x=\left(x_{1}, 0, \ldots, 0, x_{m+1}, \ldots, x_{n}\right)$ so that $q(x)=x_{1}^{2}-x_{m+1}^{2}-\cdots-x_{n}^{2}>0$ and $x_{1}>0$, namely it is the forward light cone.

Observe that with our normalization of the Jordan triple system, we have $\|x\|^{2}=$ $2\left(x_{1}^{2}+\cdots+x_{n}^{2}\right)$. Correspondingly $d x=\sqrt{2}^{n} d x_{1} \ldots d x_{n}$. Also the genus $p=n$. The integral $I(\nu, \underline{\alpha})$ is, after the change of variables as in Section 3,

$$
I(\nu, \underline{\lambda})=\int_{\Omega} \Delta_{\alpha}(\xi) \Delta(\xi)^{\frac{\nu}{2}-\frac{n}{2}}\left(\int_{B} \frac{1}{\Delta(e+\xi-y)^{\nu}} d y\right) d \xi
$$


The integration on $B$ can be evaluated directly. Observe that $\Delta(x-y)=\Delta(x)+$ $\Delta(y)$ if $x+y \in A \oplus B$. Performing a change of variable $\eta=\Delta(e+\xi)^{-\frac{1}{2}} y$ we have

$$
\begin{aligned}
\int_{B} & \frac{1}{\Delta(e+\xi-y)^{\nu}} d y=\Delta(e+\xi)^{-\nu+\frac{m-1}{2}} \int_{B} \frac{1}{\Delta(e-\eta)^{\nu}} d \eta \\
& =\sqrt{2}^{m-1} \Delta(e+\xi)^{-\nu+\frac{m-1}{2}} \int_{\mathbb{R}^{m-1}} \frac{1}{\left(1+\eta_{2}^{2}+\cdots+\eta_{m}^{2}\right)^{\nu}} d \eta_{2} \ldots d \eta_{m} \\
& =\sqrt{2 \pi}^{m-1} \frac{\Gamma\left(\nu-\frac{m-1}{2}\right)}{\Gamma(\nu)} \Delta(e+\xi)^{-\nu+\frac{m-1}{2}}
\end{aligned}
$$

Eventually we find that

$$
\begin{aligned}
I(\nu, \underline{\lambda}) & =\sqrt{2 \pi}^{m-1} \frac{\Gamma\left(\nu-\frac{m-1}{2}\right)}{\Gamma(\nu)} \int_{\Omega} \Delta_{\alpha}(\xi) \Delta(\xi)^{\frac{\nu}{2}-\frac{n}{2}} \Delta(e+\xi)^{-\nu+\frac{m-1}{2}} d \xi \\
& =\sqrt{2 \pi}^{m-1} \frac{\Gamma\left(\nu-\frac{m-1}{2}\right)}{\Gamma(\nu)} \frac{\Gamma_{\Omega}\left(\alpha+\frac{\nu}{2}-\frac{n}{2}+\frac{n-m+1}{2}\right) \Gamma_{\Omega}\left(\alpha+\frac{\nu}{2}+\frac{n}{2}-\frac{n-m+1}{2}\right.}{\Gamma_{\Omega}\left(\nu-\frac{m-1}{2}\right)}
\end{aligned}
$$

where the Gamma function $\Gamma_{\Omega}$ in this case is

$$
\Gamma_{\Omega}(\beta)=\sqrt{2 \pi}^{n-m-1} \prod_{j=1}^{2} \Gamma\left(\beta_{j}-\frac{n-m-1}{2}(j-1)\right) .
$$

Proposition 5.2. Suppose $\nu>p-2=2 p_{0}-2$,

$$
-\frac{1}{2}(\nu-m+1)<\alpha_{1}<\frac{1}{2}(\nu-n+2 m+2)
$$

and

$$
-\frac{1}{2}(\nu-n)<\alpha_{2}<\frac{1}{2}(\nu+m-1) .
$$

The integral $I(\nu, \underline{\alpha})$ is absolutely convergent and its value is given by

$$
I(\nu, \underline{\lambda})=\sqrt{2 \pi}^{m-1} \frac{\Gamma\left(\nu-\frac{m-1}{2}\right)}{\Gamma(\nu)} \frac{\Gamma_{\Omega}\left(\alpha+\frac{\nu}{2}-\frac{m-1}{2}\right) \Gamma_{\Omega}\left(\underline{\alpha}^{*}+\frac{\nu}{2}+\frac{m-1}{2}\right)}{\Gamma_{\Omega}\left(\nu-\frac{m-1}{2}\right)} .
$$

6. The $L^{p}$-BOUnded PROPERTIES OF THE BEREZIN TRANSForm

We let $C_{\nu}=I(\nu, 0)^{-1}$, which is the normalization constant. Denote

$$
b_{\nu}(\underline{\lambda})=C_{\nu} I(\nu, \underline{\alpha}) \text {. }
$$

Recall the definition of the genus $p_{0}$ of $D$. We see that, after simplifying,

$$
b_{\nu}(\underline{\lambda})=\frac{\prod_{j=1}^{r} \Gamma\left(\nu-\frac{p_{0}-1}{2}+\lambda_{j}\right) \Gamma\left(\nu-\frac{p_{0}-1}{2}-\lambda_{j}\right)}{\prod_{j=1}^{r} \Gamma\left(\nu-\frac{p_{0}-1}{2}+\rho_{j}\right) \Gamma\left(\nu-\frac{p_{0}-1}{2}-\rho_{j}\right)}
$$

if it is of type $B C$ or $C$,

$$
b_{\nu}(\underline{\lambda})=\frac{\prod_{j=1}^{r} \Gamma\left(\frac{\nu}{2}-\frac{p_{0}-1}{2}+\lambda_{j}\right) \Gamma\left(\frac{\nu}{2}-\frac{p_{0}-1}{2}-\lambda_{j}\right)}{\prod_{j=1}^{r} \Gamma\left(\frac{\nu}{2}-\frac{p_{0}-1}{2}+\rho_{j}\right) \Gamma\left(\frac{\nu}{2}-\frac{p_{0}-1}{2}-\rho_{j}\right)}
$$

for other types except $D_{2}$. So that $b_{\nu}(\underline{\lambda})$ has a rather general form in terms of $p_{0}$. For type $D_{2}$ it is

$$
b_{\nu}(\underline{\lambda})=\frac{\prod_{j=1}^{2} \Gamma\left(\frac{\nu}{2}-\frac{n-2}{4}+\lambda_{j}\right) \Gamma\left(\frac{\nu}{2}-\frac{n-2 m}{4}-\lambda_{j}\right)}{\prod_{j=1}^{2} \Gamma\left(\frac{\nu}{2}-\frac{n-2}{4}+\rho_{j}\right) \Gamma\left(\frac{\nu}{2}-\frac{n-2 m}{4}-\rho_{j}\right)}
$$


Proposition 6.1. Suppose $\nu>p-1=2 p_{0}-1$ for type $B C$ or $C$ and $\frac{\nu}{2}>p_{0}-1$ for other types. The Berezin transform $B_{\nu}$ defines a bounded positive operator on $L^{p}\left(D, d \mu_{0}\right)$ for all $1 \leq p \leq \infty$.

We recall that the functions $e_{\underline{\lambda}}$ transform under a character of $N A$. Observe that by a change of variables in the formula $B_{\nu} e_{\underline{\lambda}}(z)=b_{\nu}(\underline{\lambda}) e_{\underline{\lambda}}(z)$ for $z=e$ one obtains

$$
B_{\nu} e_{\underline{\lambda}}(z)=b_{\nu}(\underline{\lambda}) e_{\underline{\lambda}}(z)
$$

for all $z \in \mathcal{S}$; see $[1$.

In particular, taking $\underline{\lambda}+\underline{\rho}=0$ we get

$$
C_{\nu} \int_{\mathcal{S}} \frac{\Delta(\Re z)^{\frac{\nu}{2}} \Delta(\Re w)^{\frac{\nu}{2}}}{\Delta\left(\frac{1}{2}\left(z_{2}+w_{2}^{*}\right)-\frac{1}{2} F\left(z_{1}, w_{1}\right)\right)^{\nu}} d \mu_{0}(w)=1 .
$$

Proof. We use interpolation. For $p=1$ we have, in view of (6.2),

$$
\begin{aligned}
\left\|B_{\nu} f\right\|_{L^{1}} & \leq C_{\nu} \int_{\mathcal{S}} \int_{\mathcal{S}}|f(w)| \frac{\Delta(\Re z)^{\frac{\nu}{2}} \Delta(\Re w)^{\frac{\nu}{2}}}{\Delta\left(\frac{1}{2}\left(z_{2}+w_{2}^{*}\right)-\frac{1}{2} F\left(z_{1}, w_{1}\right)\right)^{\nu}} d \mu_{0}(w) d \mu_{0}(z) \\
& =\int_{\mathcal{S}}|f(w)|\left(C_{\nu} \int_{\mathcal{S}} \frac{\Delta(\Re z)^{\frac{\nu}{2}} \Delta(\Re w)^{\frac{\nu}{2}}}{\Delta\left(\frac{1}{2}\left(z_{2}+w_{2}^{*}\right)-\frac{1}{2} F\left(z_{1}, w_{1}\right)\right)^{\nu}} d \mu_{0}(z)\right) d \mu_{0}(w) \\
& =\int_{\mathcal{S}}|f(w)| d \mu_{0}(w)=\|f\|_{L^{1}},
\end{aligned}
$$

that is, $B_{\nu}$ is $L^{1}$-bounded. However, $B_{\nu}$ is a formally self-adjoint operator, thus it is $L^{\infty}$ bounded. By interpolation we see that it is bounded on all $L^{p}$, for $1 \leq p \leq$ $\infty$.

We summarize our main results in the following

Theorem 6.2. Suppose $\nu>p-1=2 p_{0}-1$ for type $B C$ or $C$ and $\frac{\nu}{2}>p_{0}-1$ for other types. The Berezin transform $B_{\nu}$ is a positive bounded operator on $L^{2}\left(D, d \mu_{0}\right)$ and its spectral symbol is given by $b_{\nu}(\underline{\lambda})$ under the decomposition of $L^{2}$-space into irreducible representations of $G$.

Remark 6.3. Recall the definition (1.1) of the restriction operator $R$. The operator $B_{\nu}=R R^{*}$ is a bounded operator, which in turn implies that $R$ is bounded when $\nu$ satisfies the condition above.

There are various implications of our results. We plan to pursue them in the future.

\section{REFERENCES}

1. J. Arazy and G. Zhang, $L^{q}$-estimates of spherical functions and mean-value property, Integral Equations and Operator Theory, 23 (1995), 123-144. MR 96g:22015

2. F. A. Berezin, General concept of quantization, Commun. Math. Phys. 40 (1975), 153-174. MR 53:15186

3. J. Faraut and A. Koranyi, Analysis on symmetric cones, Oxford University Press, Oxford, 1994. MR 98g:17031

4. S. Helgason, Differential geometry and symmetric spaces, Academic Press, New York, London, 1978. MR 80k:53081

5. J. Hilgert and G. Ólafsson, Causal symmetric spaces, geometry and harmonic analysis, Perspectives in Mathematics, vol. 18, Academic Press, 1997. MR 97m:43006

6. S. C. Hille, Canonical representations, Ph.D. thesis, Leiden University, 1999. 
7. L. K. Hua, Harmonic analysis of functions of several complex variables in the classical domains, Amer. Math. Soc., Providence, Rhode Island, 1963. MR 30:2162

8. B. Kostant and S. Sahi, Jordan algebras and Capelli identities, Invent. Math. 112 (1993), 657-664. MR 94b:17054

9. O. Loos, Bounded symmetric domains and Jordan pairs, University of California, Irvine, 1977.

10. Yu. Neretin, Matrix analogs of the integral $B(\alpha, \rho-\alpha)$ and Plancherel formula for Berezin kernel representations, (1999), preprint, Math.RT/9905045.

11. G. Ólafsson, Causal symmetric spaces, Mathematica Gottingensis 15 (1990).

12. G. Ólafsson and B. Ørsted, Generalizations of the Bargmann transform, Lie theory and its applications in physics. Proceedings of the international workshop, Clausthal, Germany, August 14-17, 1995. (H.-D.Doebner et al, ed.), World Scientific, Singapore, 1996, pp. 3-14. MR 99e:22032

13. B. Ørsted and G. Zhang, Weyl quantization and tensor products of Fock and Bergman spaces, Indiana Univ. Math. J. 43 (1994), 551-582. MR 95h:22008

14.,$L^{2}$-versions of the Howe correspondence I, Math. Scand. 80 (1997), 125-160. MR 99c:22017

15. J. Peetre, Berezin transform and Ha-plitz operators, J. Oper. Theory 24 (1990), 165-168. MR 91k:47058

16. G. Shimura, Generalized Bessel functions on symmetric spaces, J. Reine Angew. Math. 509 (1999), 35-66. MR 2000e:33020

17. A. Unterberger and H. Upmeier, The Berezin transform and invariant differential operators, Comm. Math. Phys. 164 (1994), 563-597. MR 96h:58170

18. G. van Dijk and S. C. Hille, Canonical representations related to hyperbolic spaces, J. Funct. Anal. 147 (1997), 109-139. MR 98k:22053

19. G. van Dijk and M. Pevzner, Berezin kernels and tube domains, J. Funct. Anal., to appear.

20. A. M. Vershik, I.M. Gel'fand, and M.I. Graev, Representations of the group $S L(2, \mathbf{R})$ where $\mathbf{R}$ is a ring of functions, Uspekhi Mat. Nauk 28 (1973), no. 5, 83-128.

21. G. Zhang, Berezin transform on line bundles over bounded symmetric domains, J. Lie Theory 10 (2000), 111-126. MR 2001c:32015

Department of Mathematics, Chalmers University of Technology and Göteborg UniVERSity, S-412 96 GÖteborg, Sweden

E-mail address: genkai@math.chalmers.se 